\title{
Risk Factors for Adverse Cardiac Events After Lumbar Spine Fusion
}

\author{
I. DAVID KAYE, MD, SCOTT C. WAGNER, MD, JOSEPH S. BUTLER, MD, ARJUN SEBASTIAN, MD, \\ PATRICK B. MORRISSEY, MD, CHRISTOPHER KEPLER, MD \\ Rothman Institute at Thomas Jefferson University Hospital, Philadelphia, Pennsylvania
}

\begin{abstract}
Background: To determine the incidence and risk factors for adverse cardiac events after lumbar spine fusion. Methods: A total of 50495 patients were identified through the American College of Surgeons National Surgical Quality Improvement Program (ACS-NSQIP) database who underwent lumbar spine fusion between 2005 and 2015. The 30-day postoperative data were analyzed to assess for the incidence of adverse cardiac events including cardiac arrest or myocardial infarction. Of those who experienced an event, patient- and surgery-specific parameters were evaluated to assess for risk factors.

Results: A total of 240 cardiac events occurred in the studied cohort ( 4.76 events/1000 patients). Factors that were associated with an increased cardiac risk were age (odds ratio $[\mathrm{OR}]=1.039,95 \%$ confidence interval $[\mathrm{CI}]=1.03,1.05, P$ $<.001)$, male sex $(\mathrm{OR}=1.51,95 \% \mathrm{CI}=1.17,1.94, P=.001)$, insulin-dependent diabetes $(\mathrm{OR}=1.83,95 \% \mathrm{CI}=1.29,2.6$, $P=.001)$, American Society of Anesthesiologists (ASA) score $>3(\mathrm{OR}=1.92,95 \% \mathrm{CI}=1.00,3.65, P=.048)$, absolute hematocrit different from $45(\mathrm{OR}=1.07,95 \% \mathrm{CI}=1.04,1.10, P<.001)$, and smoking $(\mathrm{OR}=1.39,95 \% \mathrm{CI}=1.02,1.90$, $P=.04)$. The impact of sustaining a cardiac event in the setting of single-level lumbar fusion is catastrophic as the 30-day postoperative mortality rate for those sustaining an event was $24.6 \%$ (59/240 patients), compared to $0.2 \%$ (87/50 255) for those not sustaining an event $(P<.001)$.

Conclusions: Cardiac events after lumbar fusion are a rare but devastating series of complications. Several risk factors were identified, including insulin-dependent diabetes mellitus, smoking, advanced age, male sex, ASA score of $>3$, and anemia/polycythemia. Considering the severity of these consequences, appropriate risk stratification is imperative, and optimization of modifiable risk factors may mitigate this risk.
\end{abstract}

Lumbar Spine

Keywords: lumbar fusion, adverse cardiac event, ACS-NSQIP database, risk factors

\section{INTRODUCTION}

Lumbar fusion is increasingly performed as emerging data continue to confirm its benefit in the setting of spinal stenosis accompanied by instability, especially in cases of spondylolisthesis. ${ }^{1,2}$ Moreover, as life expectancies increase, these procedures are being performed on an even older population. Although excellent outcomes have been established for properly indicated patients undergoing lumbar fusion, the aging patient population for many of these procedures often carries multiple comorbidities. In part because of associated comorbidities, complication rates for lumbar fusion are upwards of $25 \% .^{3}$

Cardiac events, the leading cause of mortality after noncardiac surgery, ${ }^{4,5}$ are an especially nefarious group of complications with a reported range from $0.01-13 \%^{5-8}$ after lumbar fusion. Lee et $\mathrm{al}^{7}$ found that patients who had sustained a cardiac event after spine surgery had an increased 2-year mortality compared to those without a perioperative cardiac event.

Risk stratification prior to lumbar spine surgery can mitigate the untoward consequences of perioperative complications, but accurate assessment of these risk factors is paramount. ${ }^{9}$ Although several studies have evaluated the incidence and morbidity of complications after lumbar spine surgery, fewer have attempted to identify risk factors for the development of specific medical conditions, such as risk factors for pneumonia after lumbar fusion. ${ }^{10}$ Only 1 study has specifically evaluated risk factors for cardiac events after lumbar fusion surgery. ${ }^{5}$ However, that study was performed as a relatively small, single-institution retrospective review in a South Korean population. 
Table 1. Variables included in logistic regression.

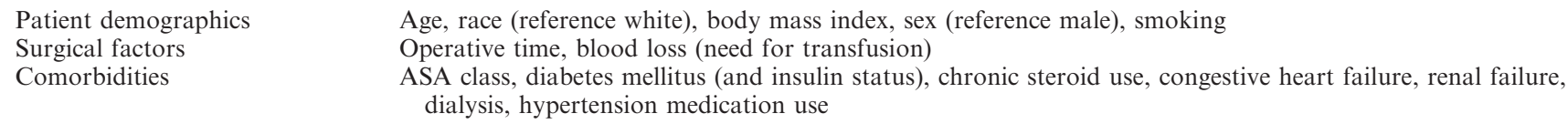

Abbreviation: ASA, American Society of Anesthesiologists.

Accurate assessment of cardiac risk is essential for predicting potential complications and attempting to medically optimize certain conditions prior to surgery. Moreover, it allows for a more meaningful informed consent process for patients deliberating spinal fusion.

Over the past several years, database studies have become increasingly used to study infrequent events through inclusion of large patient numbers, allowing evaluation of rare complications across a heterogeneous population. The American College of Surgeons National Surgical Quality Improvement Program (ACS-NSQIP) is a database that prospectively collects data on thousands of patients being treated at participating institutions across the country and includes surgeon input data on 30-day perioperative complications.

The purpose of this study is to use the ACSNSQIP database to assess risk factors for cardiac events after lumbar spine surgery.

\section{MATERIALS AND METHODS}

The ACS-NSQIP was used to analyze risk factors for the development of cardiac events after singlelevel lumbar spine fusion. The ACS-NSQIP is a surgical registry that prospectively collects 30 -day postoperative data, including complications such as myocardial infarction, sepsis, and death, from numerous participating institutions across the United States.

\section{Data Collection}

A total of 50495 patients who underwent lumbar spine fusion between 2005 and 2015 were included. Patients with the following current procedural terminology (CPT) codes were included for study: 22612 (arthrodesis, posterior or posterolateral technique, single level), 22558 (arthrodesis, anterior interbody technique, including minimal discectomy to prepare interspace [other than for decompression]), 22630 (arthrodesis, posterior interbody technique, including laminectomy and/or discectomy to prepare interspace [other than for decompression]), and 22633 (arthrodesis, combined posterior or posterolateral technique with posterior interbody technique including laminectomy and/or discectomy sufficient to prepare interspace [other than for decompression], single interspace and segment; lumbar). The ACS-NSQIP database excludes pediatric cases, and therefore, children were not included in this study. Patients were stratified based on whether or not they experienced an adverse cardiac event, either a cardiac arrest or myocardial infarction. To be diagnosed with a cardiac event according to NSQIP guidelines, there must be documentation of elevated troponins and an altered electrocardiography. Other cardiac events were not evaluated, as they are not included in the NSQIP database.

The 2 cohorts, with and without a cardiac event, were then evaluated, and demographics, comorbidities, and intra-operative factors were compared to assess for risk factors for adverse events. Demographic factors included age, sex, race, body mass index, and current smoking status. Comorbidity factors included American Society of Anesthesiologists (ASA) class, diabetes mellitus (and insulin status), chronic steroid use, congestive heart failure, renal failure, dialysis, peripheral vascular disease, chronic obstructive pulmonary disease, preoperative hematocrit (Hct), and hypertension. Operative characteristics included operative duration and blood loss (Table 1).

\section{STATISTICAL METHODS}

Statistical analysis was performed using the rms package for $\mathrm{R}$ ( $\mathrm{R}$ Foundation for Statistical Computing, Vienna, Austria). Continuous variables were compared using Mann-Whitney tests, while all others were compared with Fisher's exact test. ASA class, as a ranked variable, was examined with Mann-Whitney test.

We had assumed that, given the rarity of sustaining an adverse cardiac event in the perioperative period after lumbar spine fusion, the compared cohorts would be relatively disparate in terms of size (ie, hundreds versus thousands). We have used a logistic regression, and generally, there should be 
Table 2. Multivariate analysis of risk factors.

\begin{tabular}{|c|c|c|c|}
\hline & Odds Ratio & 95\% (Confidence Interval) & $P$ Value \\
\hline Age, y & 1.04 & $1.03-1.05$ & $<.001$ \\
\hline ASA 2 versus 1 & 0.83 & $0.46-1.51$ & .546 \\
\hline ASA 3 versus 1 & 1.28 & $0.71-2.30$ & .417 \\
\hline ASA 4 versus 1 & 1.92 & $1.00-3.65$ & .048 \\
\hline Diabetes mellitus insulin versus none & 1.83 & $1.29-2.59$ & .001 \\
\hline Noninsulin & 1.13 & $0.08-1.58$ & .488 \\
\hline Oral & 1.23 & $0.65-2.31$ & .531 \\
\hline Smoker & 1.39 & $1.02-1.90$ & .040 \\
\hline Male versus female & 1.51 & $1.17-1.94$ & .001 \\
\hline Absolute difference in hematocrit from 45 (per point) & 1.07 & $1.04-1.10$ & $<.001$ \\
\hline
\end{tabular}

Abbreviation: ASA, American Society of Anesthesiologists.

10-20 events (ie, cardiac events) per predictor for this type of analysis to be trustworthy. In the current study, the frequency of events (on the order of hundreds of patients sustaining a cardiac event) would be adequate for 12-24 predictors. Moreover, multivariate regression modeling was created using penalized logistic regression to keep the model from overfitting. ${ }^{11}$ Certain variables, such as body mass index (BMI), were pruned as they did not improve model performance as indicated by the Akaike information criterion. ${ }^{11}$ The level of significance was set at $P<.05$. Ultimately, even with disparate sample sizes, we have used tools to ensure that the results are statistically meaningful and to limit undue optimism.

\section{RESULTS}

A total of 240 cardiac events occurred among 50495 patients undergoing lumbar spine fusion between 2005 and 2015 (4.76 events per 1000 patients). In the multivariate analysis (Table 2), factors that were associated with an increased cardiac risk were age (odds ratio $(\mathrm{OR})=1.039$, $95 \%$ confidence interval $(\mathrm{CI})=1.03,1.05, P<.001)$, male sex $(\mathrm{OR}=1.51,95 \% \mathrm{CI}=1.17,1.94, P=.001)$, insulin-dependent diabetes $(\mathrm{OR}=1.83,95 \% \mathrm{CI}=$ $1.29,2.6, P=.001)$, ASA score of 4 versus a score of $1(\mathrm{OR}=1.92,95 \% \mathrm{CI}=1.00,3.65, P=.048)$, absolute Hct different from $45(\mathrm{OR}=1.07,95 \% \mathrm{CI}$ $=1.04,1.10, P<.001)$, and smoking $(\mathrm{OR}=1.39$, $95 \% \mathrm{CI}=1.02,1.90, P=.04)$.

Characteristics that were not associated with cardiac events included race, BMI, noninsulindependent diabetes, ASA class $<4$, and several other comorbidities.

Older patients were more likely to experience a cardiac event with the average age of those sustaining a cardiac event of 66.75 years $( \pm 10.52$ years) compared to those without a cardiac event whose average age was 59.26 years $( \pm 13.58$ years; $P$ $<.001)$. Each increasing year above 65 portends a $3 \%$ increased cardiac risk.

Plotting of the data indicated that preoperative Hct had a U-shaped or parabolic relationship, so for simplicity and clarity, absolute differences from a Hct of 45 was used in the model (ie, a Hct of 40 and 50 both have values of 5). The average preoperative Hct of those with a cardiac event was $38.89( \pm 6.03)$ compared to $40.69( \pm 4.41)$ for those without an event $(P<.001)$. For each point change in Hct from 45 , there is a $7 \%$ increased risk of an event.

Regarding comorbidities, $15.4 \%$ of those sustaining a cardiac event had insulin-dependent diabetes compared to only $5.5 \%$ of those without an event ( $P$ $=.001)$. Insulin-dependent diabetes increased the risk of a cardiac event by $80 \%$. Being insulin dependent was found to be equivalent to increasing age by 16 years or by altering the preoperative Hct by 8.5 points.

The impact of sustaining a cardiac event in the setting of single-level lumbar fusion is catastrophic as the 30 -day postoperative mortality rate for those sustaining an event was $24.6 \%$ (59/240 patients), compared to $0.2 \%(87 / 50255)$ for those not sustaining an event $(P<.001)$.

\section{DISCUSSION}

Cardiac events after lumbar fusion are rare but have devastating complications. Reviewing a 10year period captured by the NSQIP database, the risk of sustaining a cardiac event after lumbar spine fusion was $4.76 / 1000$ cases. The risk of mortality among that cohort was an alarmingly high $24.6 \%$, which mirrors the mortality risk identified by Fineberg et $\mathrm{al}^{6}$ in a similar cohort in their analysis of the National Inpatient Sample (NIS) database for patients undergoing lumbar fusion. This staggering increase in mortality highlights the importance of 
identifying patient risk factors and ensuring medical optimization prior to lumbar surgery.

This study identified several risk factors which increased the chances of sustaining a cardiac complication after single-level lumbar fusion. Specifically, insulin-dependent diabetes mellitus, smoking, advanced age, male sex, ASA score $>3$, and a preoperative Hct different from 45 all increased risk.

Other studies have also identified some of these risk factors which can increase complication rates in general after spine surgery. Grusakay et al ${ }^{12}$ reviewed the NSQIP database to identify risk factors for complications and length of stay after anterior cervical discectomy and fusion. Male sex, age over 65 years, preoperative anemia, and ASA $>3$ were all predictive of major complications. Increasing age, identified as a risk factor for adverse cardiac events, has been repeatedly found to increase risk after spine surgery. ${ }^{6,13-15}$ These patients likely have increased comorbidities compounding their inherent risk, but even when those were controlled for in the current regression analysis, increasing age was associated with a $3 \%$ increased cardiac risk for each year above 65 years.

Fineberg et $\mathrm{al}^{6}$ reviewed the NIS database to assess the incidence and mortality of cardiac events after lumbar spine surgery and found that older patients were more likely to experience cardiac complications after lumbar surgery, with a 9.4-year difference between those sustaining a cardiac event and those who did not (64.5 years versus 55.1 years, $\mathrm{OR}=2.2, P<.0005)$.

Insulin resistance and dependence have been associated with increased mortality and risk for adverse cardiovascular events. ${ }^{16-18}$ Insulin dependence may be a proxy for advanced disease, reflecting progressive macro and microvascular disease, including coronary artery disease, atherosclerosis, and diabetic cardiomyopathy. In addition to increased cardiovascular risk from the pathophysiology of the disease, several studies have also revealed that the biological effects of insulin themselves are both proatherosclerotic and antiatherosclerotic in which the net balance is unknown but may lead to progressive cardiovascular disease. In our study, insulin-dependent diabetes was equivalent, in terms of cardiac risk, to being 16 years older or having an 8.5-point change in Hct.

Several studies have examined the effects of insulin dependence on postsurgical complications after spine surgery. Liu et $\mathrm{l}^{19}$ performed a meta-analysis comparing the surgical outcomes after cervical spondylotic myelopathy for those with and without diabetes. Diabetic patients had a significantly increased risk of cardiac complication after cervical spine surgery. Guzman et $\mathrm{al}^{20}$ used the NIS database to study the effects of glycemic control on perioperative morbidity and mortality for those undergoing lumbar spine surgery. Relative to nondiabetic patients, uncontrolled diabetic patients had significantly increased risk for cardiac complications.

Using a prospectively collected database over a 1year period, Guyot et $\mathrm{al}^{21}$ attempted to determine risk factors for cardiac complications after spine surgery. They identified 136 cardiac complications in 107 patients $(6.7 \%)$. Age, diabetes, elevated Charleston comorbidity index, revision surgery, combined anterior-posterior approach, and previous cardiac history were identified as risk factors.

Our study also identified preoperative Hct as a risk factor for cardiac events. We identified a parabolic curve regarding preoperative Hct and cardiac risk such that point deviation from 45 in either direction was associated with a $7 \%$ increased risk of sustaining a cardiac event. This is particularly concerning, given that studies have found that preoperative anemia is relatively common in patients undergoing elective surgery. ${ }^{22,23}$ Although adults have a remarkable tolerance for changes in Hct through an increase in cardiac output and improved oxygen extraction, the elderly are more sensitive to these changes and less able to compensate. Surgery may further stress these compensatory mechanisms.

Preoperative anemia has been implicated as a risk factor for adverse cardiac events, increased length of stay, and mortality. ${ }^{24}$ Seicean et $\mathrm{al}^{25}$ analyzed the ACS-NSQIP database to assess whether preoperative anemia predicted adverse early perioperative outcomes in patients undergoing spine surgery. Any degree of preoperative anemia was associated with higher risk for adverse outcomes compared to no anemia. Similarly, Kim et $\mathrm{al}^{26}$ evaluated the 30-day outcomes in a cohort of VA NSQIP patients undergoing major noncardiac surgery and found that increases or decreases in preoperative Hct increased cardiac risk, with a $1.6 \%$ increased mortality for each point above $51 \%$ or below $39 \%$.

Our study also demonstrated the increased risk of sustaining an adverse cardiac event with increasing Hct. Increased Hct has been linked to an increased risk of atherosclerosis and the development of 
cardiovascular disease. ${ }^{27}$ Polycythemia can increase blood viscosity and can secondarily result in increased cardiac afterload which decreases cardiac output, leading to organ hypoperfusion. These effects can be exacerbated by surgery and lead to increased cardiovascular events, as shown by both the current study and others. ${ }^{26}$

The only 2 readily modifiable risk factors identified were preoperative Hct and smoking status, both of which should be optimized prior to surgery. Smoking is known to cause alteration in vascular hemostasis and the normal clotting cascade and can alter platelet membranes, which alters normal physiologic functioning. Martin et $\mathrm{al}^{28}$ found that current smokers had a statistically significant morbidity index, but that former smokers did not. The authors conclude that smoking cessation may mitigate the 30 -day morbidity risk after lumbar spine surgery. Our study confirms this increased risk and suggests that risk factors, such as smoking and preoperative Hct abnormalities, should ideally be modified prior to undergoing lumbar spine fusion.

\section{Limitations}

This study sought to specifically identify risk factors for cardiac events after lumbar spine fusion by reviewing a national database with data driven largely by clinician input. The robust patient-based data available in the NSQIP are both a strength and weakness of the study. Access to such a large cohort allows analysis of less frequent complications and facilitates identification of risk factors which would otherwise potentially remain undetected. However, the dataset is subject to the limitations of all database studies with incomplete data and clinician input bias. However, the NSQIP has specific guidelines for the documentation of a cardiac event, and given its gravity, the dataset for this complication alone may be more accurate than for others.

However, the data are limited to variables included in the NSQIP database and known risk factors for myocardial infarction. For instance, elevated cholesterol and triglyceride levels are missing and may have proven to be predictive of risk.

Fineberg et $\mathrm{al}^{6}$ had performed an evaluation of the NIS database to assess the incidence and mortality of cardiac events in lumbar spine fusion. We have alternatively used the NSQIP database, which provided some similar, but some different risk factors. The NIS database is an administrative dataset with complications coded by International Classification of Diseases, Ninth Revision (ICD-9) codes, while the NSQIP database is comprised of data input by surgical clinical reviewers who are nurses specially trained in data entry for this database. It was created primarily as a quality improvement tool rather than a database used for claims processing. Bohl et $\mathrm{al}^{29}$ found that, despite the fact that NIS and NSQIP each use cohorts with similar demographics, in a lumbar fusion model, the 2 databases document markedly different rates of specific comorbidities and adverse events.

Several studies have demonstrated that ICD-9 codes, subject to interhospital variation in postoperative complication reporting, may lead to underreporting of complications. ${ }^{30,31}$ In this regard, the ACS-NSQIP has been called the gold standard for current databases. ${ }^{29}$ However, in the identification of cases of acute cardiac events, the use of ICD-9 codes has been shown by some to be accurate. ${ }^{31}$

\section{CONCLUSIONS}

Cardiac events after lumbar spine surgery are a rare but devastating series of complications. We identified a rate for adverse cardiac events of 4.76/ 1000 patients. More concerning, 24.6\% of patients who sustained a cardiac event died. Considering the severity of these consequences, appropriate risk stratification is imperative. Several risk factors were identified in the review of the ACS-NSQIP database including insulin-dependent diabetes mellitus, smoking, advanced age, male sex, ASA score of $>3$, and anemia/polycythemia. Identifying higher risk patients and counseling them about potential cardiac complications is essential for informed consent and for minimizing their occurrence. Optimization of modifiable risk factors may mitigate this risk, and future studies aimed at risk stratification and modification may help elucidate the success of these interventions.

\section{REFERENCES}

1. Weinstein JN, Lurie JD, Tosteson TD, et al. Surgical compared with nonoperative treatment for lumbar degenerative spondylolisthesis. Four-year results in the Spine Patient Outcomes Research Trial (SPORT) randomized and observational cohorts. J Bone Joint Surg Am. 2009;91(6):12951304.

2. Jancuska JM, Hutzler L, Protopsaltis TS, Bendo JA, Bosco J. Utilization of lumbar spinal fusion in New York State: trends and disparities. Spine (Phila Pa 1976). 2016;41(19):1508-1514. 
3. Di Capua J1, Somani S, Kim JS, et al. Analysis of risk factors for major complications following elective posterior lumbar fusion. Spine (Phila Pa 1976). 2017;42(17):1347-1354.

4. Faciszewski T, Jensen R, Rokey R, et al. Cardiac risk stratification of patients with symptomatic spinal stenosis. Clin Orthop Relat Res. 2001;384:110-115.

5. Lee DY, Lee SH, Jang JS. Risk factors for perioperative cardiac complications after lumbar fusion surgery. Neurol Med Chir (Tokyo). 2007;47(11):495-500.

6. Fineberg SJ, Oglesby M, Patel AA, Singh K. Incidence and mortality of perioperative cardiac events in cervical spine surgery. Spine (Phila Pa 1976). 2013;38(15):1268-1274.

7. Lee MJ, Hacquebord J, Varshney A, et al. Risk factors for medical complications after lumbar spine surgery: a multivariate analysis of 767 patients. Spine (Phila Pa 1976). 2011;36(21):1801-1806.

8. Lee MJ, Konodi MA, Cizik AM, Bransford RJ, Bellabarba C, Chapman JR. Risk factors for medical complication after spine surgery: a multivariate analysis of 1,591 patients. Spine (Phila Pa 1976). 2012;12(3):197-206.

9. Carabini LM, Zeeni C, Moreland NC, et al. Predicting major adverse cardiac events in spine fusion patients: is the revised cardiac risk index sufficient? Spine (Phila Pa 1976). 2014;39(17):1441-1448.

10. Bohl DD, Mayo BC, Massel DH, et al. Incidence and risk factors for pneumonia after posterior lumbar fusion procedures: an ACS-NSQIP study. Spine (Phila Pa 1976). 2016;41(12):1058-1063.

11. Harrell FE. Regression Modeling Strategies. With Applications to Linear Models, Logistic Regression and Survival Analysis. New York: Springer; 2001.

12. Gruskay JA, Fu M, Basques BA, et al. Factors affecting length of stay and complications after elective anterior cervical discectomy and fusion: a study of 2164 patients from the ACSNSQIP database. Clin Spine Surg. 2016;29(1):E34-E42.

13. Cassinelli EH, Eubanks J, Vogt M, et al. Risk factors for the development of perioperative complications in elderly patients undergoing lumbar decompression and arthrodesis for spinal stenosis: an analysis of 166 patients. Spine (Phila Pa 1976). 2007;32(2):230-235.

14. Vitaz TW, Raque GH, Shields CB, et al. Surgical treatment of lumbar spinal stenosis in patients older than 75 years of age. J Neurosurg. 1999;91(2 Suppl):181-185.

15. Kilincer C, Steinmetz MP, Sohn MJ, et al. Effects of age on the perioperative characteristics and short-term outcome of posterior lumbar fusion surgery. $J$ Neurosurg Spine. 2005;3(1):34-39.

16. Gamble JM, Chibrikov E, Twells LT, et al. Association of insulin dosage with mortality or major adverse cardiovascular events: a retrospective cohort study. Lancet Diabetes Endocrinol. 2017;5(1):43-52.

17. Zhang PY. Cardiovascular disease in diabetes. Eur Rev Med Pharmacol Sci. 2014;18(15):2205-2214.

18. Golinvaux NS, Varthi AG, Bohl DD, et al. Complication rates following elective lumbar fusion in patients with diabetes: insulin dependence makes the difference. Spine (Phila Pa 1976). 2014;39(21):1809-1816.

19. Liu Y, Ban DX, Kan SL, Cao TW, Feng SQ. The impact of diabetes mellitus on patients undergoing cervical spondylotic myelopathy: a meta-analysis. Eur Neurol. 2017;77(1-2):105112.
20. Guzman JZ, Iatridis JC, Skovrlj B, et al. Outcomes and complications of diabetes mellitus on patients undergoing degenerative lumbar spine surgery. Spine (Phila Pa 1976). 2014;39(19):1596-1604.

21. Guyot JP, Cizik A, Bransford R, Bellabarba C, Lee MJ. Risk factors for cardiac complications after spine surgery. Evid Based Spine Care J. 2010;1(2):18-25.

22. Ania BJ, Suman VJ, Fairbanks VF, et al. Incidence of anemia in older people: an epidemiologic study in a well-defined population. J Am Geriatr Soc. 1997;45(7):825-831.

23. Goodnough LT, Vizmeg K, Sobecks R, et al. Prevalence and classification of anemia in elective orthopedic surgery patients: implications for blood conservation programs. Vox Sang. 1992;63(2):90-95.

24. Wu WC, Schifftner TL, Henderson WG, et al. Preoperative hematocrit levels and postoperative outcomes in older patients undergoing noncardiac surgery. JAMA. 2007;297(22):2481-2488.

25. Seicean A, Seicean S, Alan N, et al. Preoperative anemia and perioperative outcomes in patients who undergo elective spine surgery. Spine (Phila Pa 1976). 2013;38(15):1331-1341.

26. Kim BD, Edelstein AI, Patel A, Lovecchio F, Kim JYS. Preoperative anemia does not predict complications after single level lumbar fusion: a propensity score-matched multicenter study. Spine (Phila Pa 1976). 2014;39(23):1981-1989.

27. Carallo C, Pujia A, Irace C, et al. Whole blood viscosity and hematocrit are associated with internal carotid atherosclerosis in men. Coron Artery Dis. 1998;9(2-3):113-117.

28. Martin CT, Gao Y, Duchman K, Pugely A. The impact of current smoking and smoking cessation on short-term morbidity risk after lumbar spine surgery. Spine (Phila Pa 1976). 2016:41(7):577-584.

29. Bohl DD, Russo GS, Basques BA, et al. Variations in data collection methods between national databases affect study results: a comparison of NIS and NSQIP databases for lumbar spine fusion procedures. JBJS. 2014;96(23):193:1-7.

30. Campbell PG, Malone J, Yadla S, et al. Comparison of ICD-9 based retrospective and prospective assessments of perioperative complications: assessment of accuracy in reporting. JNS. 2011;14(1):16-22.

31. Nasser R, Yadla S, Maltenfort MG, et al. Complications in spine surgery. A review. J Neurosurg Spine. 2010:13(2);144157.

Disclosures and COI: None of the authors have any relevant financial disclosures.

Corresponding Author: I. David Kaye, MD, Rothman Institute at Thomas Jefferson University Hospital, 925 Chestnut Street, Philadelphia, PA 19107. Phone: (732) 735-8874; Email: iandavid. kaye@gmail.com.

\section{Published 15 October 2018}

This manuscript is generously published free of charge by ISASS, the International Society for the Advancement of Spine Surgery. Copyright $@ 2018$ ISASS. To see more or order reprints or permissions, see http://ijssurgery.com. 